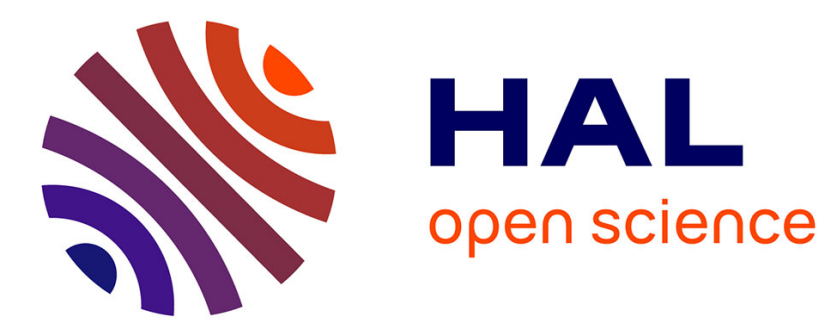

\title{
Penetration depth of a plunging jet: from microjets to cascades
}

Grégory Guyot, Alain H Cartellier, Jean-Philippe Matas

\section{To cite this version:}

Grégory Guyot, Alain H Cartellier, Jean-Philippe Matas. Penetration depth of a plunging jet: from microjets to cascades. Physical Review Letters, 2020, 10.1103/PhysRevLett.124.194503 . hal-02575121

\section{HAL Id: hal-02575121 \\ https://hal.science/hal-02575121}

Submitted on 14 May 2020

HAL is a multi-disciplinary open access archive for the deposit and dissemination of scientific research documents, whether they are published or not. The documents may come from teaching and research institutions in France or abroad, or from public or private research centers.
L'archive ouverte pluridisciplinaire HAL, est destinée au dépôt et à la diffusion de documents scientifiques de niveau recherche, publiés ou non, émanant des établissements d'enseignement et de recherche français ou étrangers, des laboratoires publics ou privés. 
Version xx as of April 2, 2020

To be submitted to (PRL)

\title{
Penetration depth of a plunging jet: from microjets to cascades
}

\author{
Grégory Guyot, ${ }^{1,2}$ Alain Cartellier, ${ }^{1}$ and Jean-Philippe Matas ${ }^{3}$ \\ ${ }^{1}$ Université Grenoble Alpes, CNRS, Grenoble-INP, LEGI, F-38000, Grenoble, France \\ ${ }^{2}$ EDF-CIH, F-73330 Le Bourget du Lac, France \\ ${ }^{3}$ Laboratoire de Mécanique des Fluides et d'Acoustique, Ecole Centrale de Lyon, \\ CNRS, Université Claude Bernard Lyon 1, INSA Lyon, F-69134 Ecully, France
}

(Dated: April 2, 2020)

\begin{abstract}
When a liquid jet impacts a pool of the same liquid, a bubble cloud is generated below the surface: we address here the question of the vertical size of this bubble cloud. We have measured this size on experiments spanning a large range of scales, from $0.3 \mathrm{~mm}$ to $210 \mathrm{~mm}$ in jet diameter, and from $0.2 \mathrm{~m}$ to $9.5 \mathrm{~m}$ in height of fall. We show that a simple model based on a balance of forces at the scale of the bubble cloud is able to account for all experimental results over this wide range of scales and types of jets. We derive from this model a simple expression for the prediction of the bubble cloud penetration depth.
\end{abstract}

Many industrial processes involve a high-speed jet plunging into a pool, and the generation of a bubble cloud below the surface. This is in particular a configuration encountered when dams spill, or in impulse turbines. This configuration happens as well in nature, for example in cascades. People have addressed the questions of bubble formation and air entrainment over a wide range of scales [1-3]: several mechanisms have been identified for explaining air entrainment, which rely on a fine modelling of the jet structure at the moment it impacts the liquid pool. Due to the industrial necessity to predict the global size of the bubble cloud, there has been a significant effort to measure the total depth $H$ of this bubble cloud for large jets (fall height of a meter or more), and to propose semi empirical or purely empirical relations to predict this quantity [4-7], but no physical model has been proposed to predict the size of the bubble cloud in these conditions. A modelling approach based on momentum conservation was successfully attempted on smaller scale jets by Clanet and Lasheras [8]: the prediction of their model is quite precise and robust for bubble clouds as small a a few millimeters up to fifteen centimeters. This model also holds when the liquid jet is oscillated in a wide range of amplitudes and frequencies, provided an effective angle of inclination (which depends on the amplitude and frequency of oscillation) is taken into account [9]. However, it fails to capture the right order of magnitude of $H$ in larger size experiments, as will be illustrated below. The aim of the present paper is to present experimental measurements of the bubble cloud size over a very wide range of scales, namely with fall heights $h$ varying from 20 centimeters to 10 meters and nozzle diameters $D$ varying from hundreds of microns up to 20 centimeters, and to propose a physical model which predicts the measured penetration depth over this wide range of scales without any fitting parameter.

Two set-ups were used in order to gather experimental data over a wide range of scales. The fluids were water and air in both set-ups. The first set-up is a cubic glass tank (edge $50 \mathrm{~cm}$ ), into which a round water jet can be issued from needles (for the smaller diameters) or a straight tube, of inner diameter $D$. The velocity at the nozzle $V_{0}$ is deduced from a flowmeter measurement. The distance $h$ between the tip of the needle and the surface of the pool is constant equal to $h=20 \mathrm{~cm}$ for this experiment. The penetration depth is visualized with a high speed camera (Phantom v10) via backlight lighting with a LED panel (see figure 1). In order to keep the water level in the pool constant, the tank is slightly tilted to the left (angle < $5^{\circ}$ ), so that it overflows over this single lateral wall. The second set-up is similar but at a much larger scale: five different nozzles with diameters ranging from $2.3 \mathrm{~cm}$ to $21.3 \mathrm{~cm}$ are used to generate the jet. A honeycomb tranquilizing chamber is included in these larger scale nozzles in order to attenuate the amplitude of secondary flows. The jet then falls over a distance $h=9.5 \mathrm{~m}$, and falls into a well of diameter $5 \mathrm{~m}$, and of a maximum depth of $23 \mathrm{~m}$ at its center. The water flows in a closed loop: two pumps ( $30 \mathrm{~kW}$ power) are used to reinject water from the well into the nozzle. The flow rate is monitored with two Krohne Optiflux electromagnetic flowmeters, one for the range $[2-50] \mathrm{m}^{3} / \mathrm{h}$ and the other for the range [50-500] $\mathrm{m}^{3} / \mathrm{h}$. The jet can be imaged during its fall by the same high speed camera used in the first experiment, which provides images of its destabilization from a round and relatively smooth coherent jet at the nozzle, to a potentially aerated flapping jet when it hits the pool. The penetration depth is measured with an underwater camera (1080 AHD Sony): the camera can be moved down to $3.5 \mathrm{~m}$ below the surface. The camera is positioned on a horizontal beam, which can be displaced vertically until the bottom of the bubble cloud coincides with the center of the field of view of the camera (figure 1). The uncertainty on the penetration depth $H$ associated with 

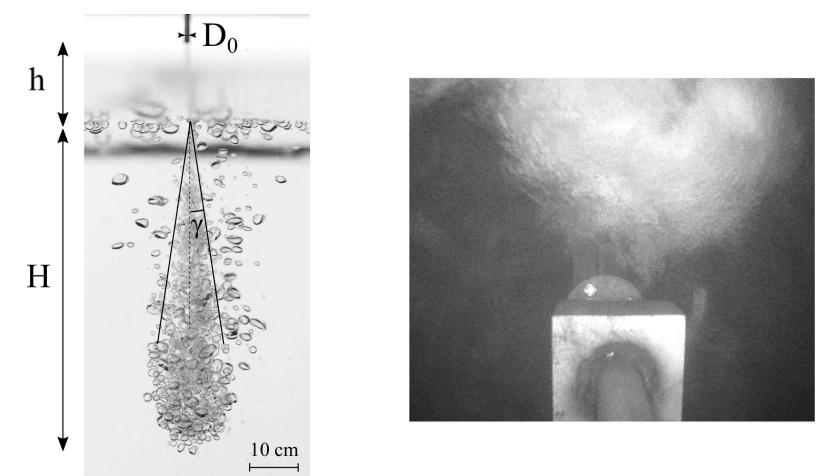

FIG. 1. Left: Example of bubble cloud in small set-up $\left(D_{0}=\right.$ $\left.1.3 \mathrm{~mm}, V_{0}=5.6 \mathrm{~m} . \mathrm{s}^{-1}\right)$. Right: Visualization of bubble cloud used for measurement of the penetration depth in large set-up $\left(D_{0}=213 \mathrm{~mm}, V_{0}=5 \mathrm{~m} . \mathrm{s}^{-1}\right)$.

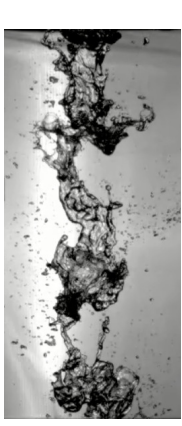

a)

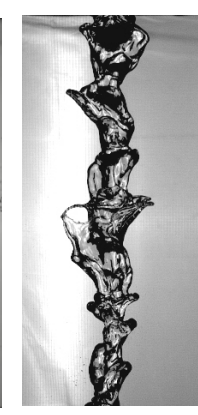

b)

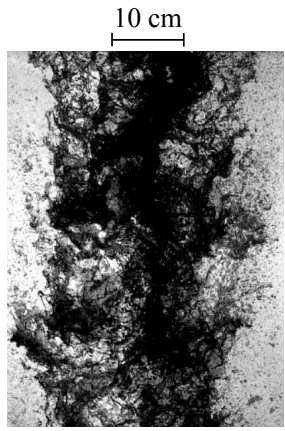

c)

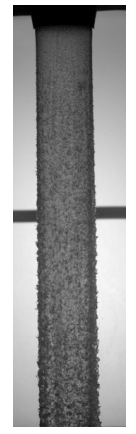

d)
FIG. 2. Illustration of the wide range of jet structures covered by our experiment: a) $D_{0}=36.4 \mathrm{~mm}$ and $V_{0}=10.6 \mathrm{~m} . \mathrm{s}^{-1}$. b) $D_{0}=82.9 \mathrm{~mm}$ and $V_{0}=3 \mathrm{~m} . \mathrm{s}^{-1}$. c) $D_{0}=82.9 \mathrm{~mm}$ and $V_{0}=23 \mathrm{~m} . \mathrm{s}^{-1} \mathrm{~d}$ ) Same conditions as c), but just after the nozzle exit. The center of images a), b) and c) is 6 meters below the nozzle.

this measurement is estimated at 10 centimeters. Figure 2 shows several examples of the jet patterns observed in our large-scale experiment. We observe a wide range of jet structures: jets which remain coherent until the impact with the pool, as in the case of the small experiment, but also jets which develop deep corrugations, flap, become aerated or end-up partially atomized after the 9.5 meters fall. High-speed videos corresponding to several typical cases are shown as supplemental material.

The penetration depths measured on both set-ups are presented in figure 3. As expected, the penetration depth increases with velocity, and it also increases with the diameter of the jet. The small scale data can be predicted by the model of Clanet and Lasheras [8], which is based on the conservation of momentum, but this model fails to capture the larger scale data, and strongly overpredicts the penetration depth: for the $D_{0}=162 \mathrm{~mm}$ series for example, it predicts values of $H$ in the range [ $4 \mathrm{~m}-10 \mathrm{~m}$ ], while experimental penetration depths vary between 2
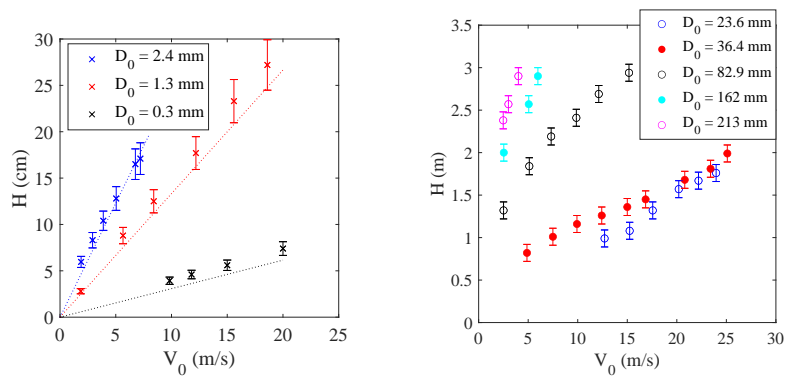

FIG. 3. Left: Penetration depth measured on the small scale set-up as a function of velocity at nozzle $V_{0}$, for various nozzle diameters $D_{0}$. The dotted line shows the prediction of the model of Clanet and Lasheras [8]. Right: Same measurement on the large scale set-up.

and 3 meters in figure 3 . The semi-empirical model of Falvey and Ervine [5], similarly overpredicts the large scale data, since it predicts $H$ in the range [7.4 m-12 m] for the same $D_{0}=162 \mathrm{~mm}$ series. The good order of magnitude of our large scale data can be captured by the purely empirical correlation proposed by McKeogh and Ervine [4], but this correlation fails in turn to capture properly the small scale data.

We model the penetration depth by writing the momentum theorem for the bubble cloud. The bubble cloud is modeled as a truncated cone, with an angle $\gamma$. Its upper section at impact is noted $S_{i}$, and we note $V_{i}$ the velocity at impact. High speed imaging of the jet before impact shows that this velocity is within $5 \%$ of $V_{i}=\sqrt{V_{0}^{2}+2 g h}$ for all the experimental conditions presented here. The bottom section of the cloud, where fluid exits the cone and bubbles reach their terminal velocity $U_{T}$, is noted $S_{T}$. The terminal velocity $U_{T}$ considered here is the maximum terminal velocity for bubbles larger than one millimeter: only submillimeter bubbles will be entrained by the liquid jet once the liquid jet velocity reaches $U_{T}$ [8]. We note $\rho_{i}$ the density of the two-phase mixture at impact, and $\rho_{T}$ the density of the mixture in the bottom section. The momentum theorem then writes

$$
\iint_{S_{i}} \rho_{i} V_{i}^{2} d S-\iint_{S_{T}} \rho_{T} U_{T}^{2} d S=F_{\text {gravity }}-F_{\text {buoyancy }}
$$

In order to model the force $F_{\text {buoyancy }}$, we assume that the void fraction $\alpha$ is constant in the bubble cloud. In particular, this implies that $\rho_{i}=\rho_{T}=\rho(1-\alpha)$. This also leads to $F_{\text {gravity }}-F_{\text {buoyancy }}=\alpha \rho g V_{\text {cone }}$ with :

$$
V_{\text {cone }}=\pi H\left(R_{i} H \tan \gamma+R_{i}^{2}+\frac{H^{2} \tan ^{2} \gamma}{3}\right)
$$

where $R_{i}$ is the radius of the jet at impact. This radius can be related to $R_{0}$ the radius at the nozzle and to the void fraction via the continuity equation: $R_{i}=$ $R_{0} \sqrt{V_{0}} / \sqrt{V_{i}(1-\alpha)}$. 


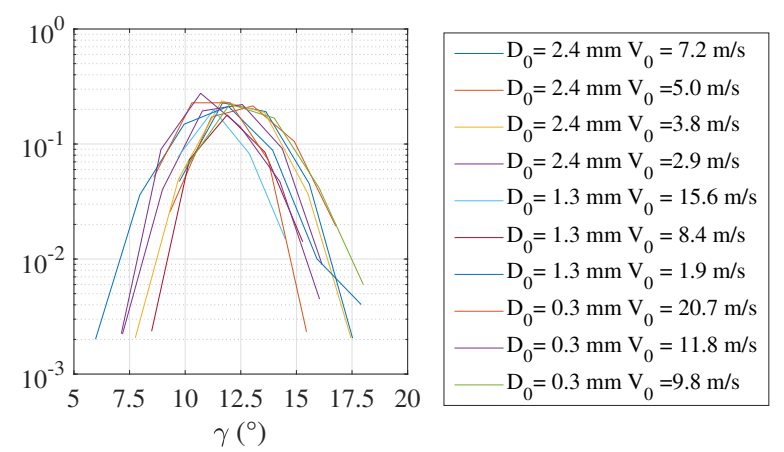

FIG. 4. Experimental probability distribution function of angle $\gamma$ values on the smaller experimental set-up: the most probable value is for all conditions close to the $\gamma=12.5^{\circ}$ value assumed by Clanet and Lasheras [8].

This finally leads to the following equation for the penetration depth $H$ :

$$
\begin{aligned}
& V_{i} V_{0} R_{0}^{2}-(1-\alpha) U_{T}^{2}\left(R_{i}+H \tan \gamma\right)^{2} \\
& \quad-\alpha g H\left(R_{i} H \tan \gamma+R_{i}^{2}+\frac{H^{2} \tan ^{2} \gamma}{3}\right)=0
\end{aligned}
$$

This equation can be solved for $H$ provided parameters $U_{T}, \gamma$ and $\alpha$ are known. The bubble terminal velocity $U_{T}$ is taken equal to $U_{T}=0.22 \mathrm{~m} / \mathrm{s}$, which is the limit value below which only submillimeter air bubbles will be entrained in water [8]. The mixing layer angle $\gamma$ can be measured on the first experimental set-up: backlight imaging measurements show that $\gamma$ values are distributed around a mean value of $12^{\circ}$ (see figure 4 ). In their momentum balance, Clanet and Lasheras [8] assume $\gamma=12.5^{\circ}$, based on well known results for turbulent single phase jets [10]. Given the weak difference between both values, we follow them and assume in the following that $\gamma$ is constant and equal to $12.5^{\circ}$ for all our experimental conditions. The impact of possible variations of $\gamma$ on the prediction of the model will be discussed further below.

The average void fraction $\alpha$ is expected to depend on the jet size and velocity, and more specifically on its dynamics during its free fall. In addition, the local void fraction varies spatially, and in particular is expected to decrease with increasing depth as water is entrained within the bubble cloud. Void fraction profiles such as those measured by McKeogh and Ervine [4] show that relatively large maximum local void fractions close to the surface and to the jet centerline (of the order of $40 \%$ to $60 \%$ ) will result into significantly lower $\alpha$ (of the order of $10 \%$ to $20 \%$ ), once averaged over the conical bubble cloud modelled here.

We plot in figure 5 the experimental penetration depth as a function of the solution of equation (1), where an average void fraction of $15 \%$ has been assumed. The model captures the correct values and scaling law for the

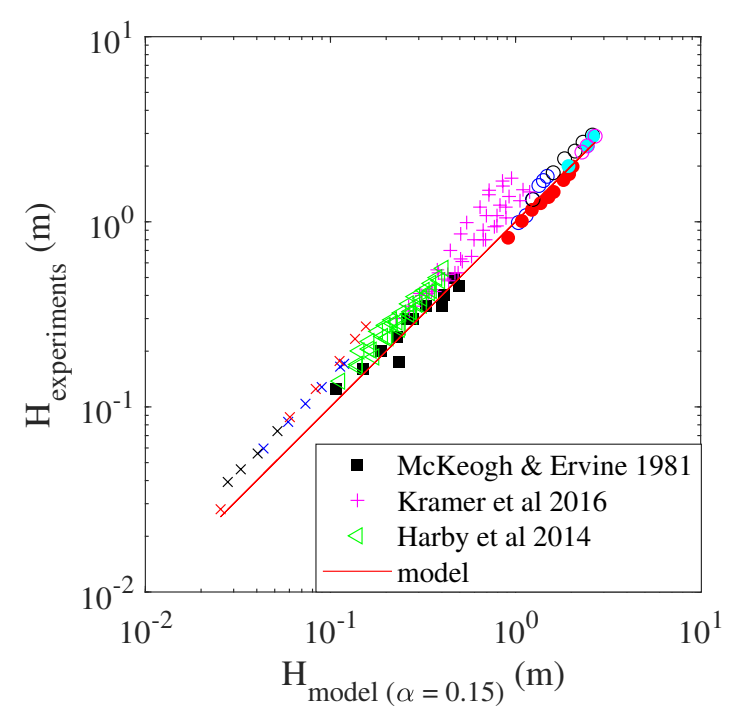

FIG. 5. Experimental penetration depth plotted as a function of the prediction of equation (1) for the case $\alpha=0.15$ : the data of the present paper (circles and crosses) follows the caption of figure 3 . The data of several other authors are included for comparison. The larger dispersion for the data of Kramer et al. [12] is due to the fact that these authors vary the void fraction.

data of both our experimental set-ups over more than two decades. We also plot in figure 5 the experimental data of several authors: the model captures very well the penetration depths measured by McKeogh and Ervine [4] and Harby et al. [11]. There is more dispersion regarding the data of Kramer et al. [12]: we attribute this dispersion to the fact that these authors have purposedly changed the fall height $h$ in their experiment, and therefore explore a wider range of void fractions $\alpha$. This is evidenced in figure $6 \mathrm{a}$, which shows a blow up of the data of these authors, where different symbols have been affected to the different heights of fall $h$. The model with $\alpha=0.15$ agrees well with the data obtained for $h=1 \mathrm{~m}$. The data corresponding to lower $h$ are underestimated by the same model: this is probably an evidence of the role of $\alpha$, which is expected to be lower at these lower $h$ due to the lesser corrugation of the plunging jet when the fall height is reduced.

One can note on figure 5 that the model slightly underestimates the penetration depth, in particular for smaller jets (symbol $\times$ ): this is probably related to the strong assumption made regarding the shape of the bubble cloud, which has been assimilated to a truncated cone. In particular the lower part of the bubble cloud is usually observed to be round (see figure 1), and not flat, due to velocity fluctuations over a scale equal to the radius of the bubble cloud at its base, namely $R_{T}=R_{i}+H \tan \gamma \approx$ $H \tan \gamma$ [8]. If we take into account this contribution in the model, the predicted value is increased by a factor 


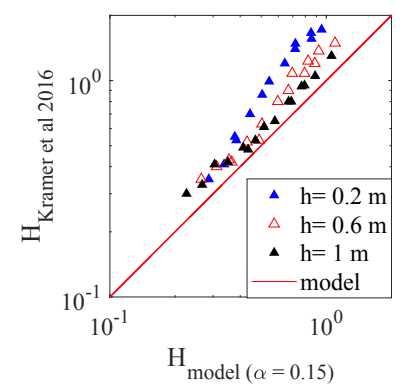

a)

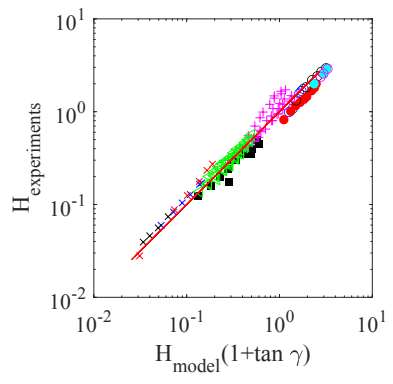

b)

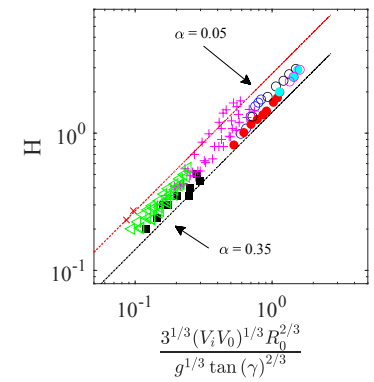

c)

FIG. 6. a) Experimental data of Kramer et al. [12]: the dispersion between their data and our model is due to the impact of the height of fall $h$, which directly impacts the void fraction $\alpha$. b) The model can be improved by introducing a $1+$ $\tan \gamma$ factor to account for velocity fluctuations at the bottom of the bubble cloud. Same caption as in figures 3 and 5. c) Illustration of the relatively weak impact of the mean void fraction $\alpha$ on the simplified prediction for the penetration depth via equation (3): predictions corresponding to $\alpha=5 \%$ and $\alpha=35 \%$ remain close to the experimental data. Only the data for which $H>H_{c}=10 \mathrm{~cm}$, i.e. for which equation (3) is valid, is represented here. The unit is meters for all axes.

$1+\tan \gamma$, and an even better agreement is found (see figure $6 \mathrm{~b})$.

Our model does not account for turbulent dissipation: in our experiments the Reynolds number of the liquid jet $R e=2 R_{i} U_{i} / \nu$ varies from 3000 (smaller jet) to $3.10^{6}$ (larger jet), and turbulence is therefore expected to vary greatly. The fact that the agreement between our model and the experimental data is good for a wide range of scales independently of these very large Re variations is an indication that the impact of turbulent dissipation can be neglected compared to the momentum loss caused by buoyancy.

In the limit of small bubble clouds, the buoyancy force becomes negligible compared to the momentum flux contribution at the bottom of the cloud, and equation (1) then simplifies into the model proposed by Clanet and Lasheras [8]. For large bubble clouds, on the contrary, the contribution due to the bottom momentum flux becomes negligible. In this limit, and assuming also that
$H \tan (\gamma) \gg R_{i}$ (lateral extent of bubble cloud large compared to jet radius at impact, which is largely true for all our data), equation (1) simplifies into:

$$
H=\frac{3^{1 / 3}\left(V_{i} V_{0}\right)^{1 / 3} R_{0}^{2 / 3}}{(\alpha g)^{1 / 3} \tan (\gamma)^{2 / 3}}
$$

This expression is independent of $U_{T}$. It predicts that $H$ scales as $V_{0}^{2 / 3} R_{0}^{2 / 3}$ when $V_{i}$ is close to $V_{0}$ : this scaling law is very similar to the popular empirical correlation proposed by McKeogh and Ervine [4], namely $H \propto V_{0}^{0.7} R_{0}^{0.7}$. The present model provides a physical basis for the relevance of this scaling law, and shows that this exponent $2 / 3$ results from the balance between a surface effect (momentum at impact) and a volume effect (net buoyancy). Equation (2) can be rewritten in dimensionless form as:

$$
\frac{H}{R_{0}}=\frac{3^{1 / 3}}{\alpha^{1 / 3} \tan (\gamma)^{2 / 3}}\left(\frac{V_{i} V_{0}}{g R_{0}}\right)^{1 / 3}
$$

This expression shows that the dimensionless penetration depth is a function of three dimensionless parameters: the angle $\gamma$ of the mixing layer (which can be safely supposed to be constant $[8,10]$ ), the Froude number, and the void fraction. Note that the exponent $1 / 3$ for the void fraction in equation (3) limits the impact of void fraction variations, and explains the robustness of the model in figure 5: even though a precise adjustment of the void fraction for each specific case would definitely increase the precision of the prediction, taking a single value of $\alpha$ for all experiments provides a surprisingly good agreement for all experimental data. This is illustrated in figure $6 \mathrm{c}$, which shows that the predictions corresponding to respectively much smaller and larger average void fractions of $5 \%$ and $35 \%$ still remain relatively close to the experimental data. Similarly, in the scaling law of equation (3) the penetration depth $H$ varies as $\tan (\gamma)^{2 / 3}$, and a given relative error on the mixing layer angle $\gamma$ therefore results in a smaller relative error in $H$.

The critical cloud size $H_{c}$ for which both momentum flux out of the cloud and buoyancy effects balance corresponds to $H_{c}=3 U_{T}^{2}(1-\alpha) /(\alpha g)$. For the case of air and water, and an average void fraction of $\alpha=0.15$, this critical size is of the order of $H_{c}=10 \mathrm{~cm}$. This indicates that for all large scale applications, and in particular applications related to hydraulics, equation (3) can be safely used to estimate the penetration depth of plunging jets.

As stated above, the accuracy of the present model could be improved with a more precise estimate of the average void fraction $\alpha$ : the modelling of air entrainment into the bubble cloud, which is in turn related to the dynamics of the falling jet and its state at impact, is therefore one of the main companion questions to address in future work. 
[1] E. Lorenceau, D. Quéré, and J. Eggers, Air entrainment by a viscous jet plunging into a bath, Phys. Rev. Lett. 93 (2004).

[2] A. K. Biń, Gas entrainment by plunging liquid jets, Chem. Eng. Sci. 48, 3585 (1993).

[3] Y. Zhu, H. N. Oğuz, and A. Prosperetti, On the mechanism of air entrainment by liquid jets at a free surface, J. Fluid Mech. 404, 151 (2000).

[4] E. J. McKeogh and D. A. Ervine, Air entrainment rate and diffusion pattern of plunging liquid jets, Chem. Eng. Sci. 36, 1161 (1981).

[5] H. T. Falvey and D. A. Ervine, Behaviour of turbulent water jets in the atmosphere and in plunge pools, ICE Proc. 83, 295 (1987).

[6] H. Nakasone, Study of aeration at weirs and cascades, J. Environ. Eng. 113, 64 (1987).

[7] A. Ohkawa, D. Kusabiraki, Y. Shiokawa, N. Sakai, and
M. Fujii, Flow and oxygen transfer in a plunging water jet system using inclined short nozzles and performance characteristics of its system in aerobic treatment of wastewater, Biotechnology and Bioengineering 28, 1845 (1986).

[8] C. Clanet and J. C. Lasheras, Depth of penetration of bubbles entrained by a plunging water jet, Phys. Fluids 9, 1864 (1997).

[9] G. Guyot, A. Cartellier, and J.-P. Matas, Depth of penetration of bubbles entrained by an oscillated plunging water jet, Chem. Eng. Science X 2, doi.org/10.1016/j.cesx.2019.100017 (2019).

[10] G. Horn and M. Thiring, Angle of spread of free jets, Nature 178, 205 (1956).

[11] K. Harby, S. Chiva, and J. Muñoz Cobo, An experimental study on bubble entrainment and flow characteristics of vertical plunging water jets, Exp. Therm. Fluid Sci. 57, 207 (2014).

[12] M. Kramer, S. Wieprecht, and K. Terheiden, Penetration depth of plunging liquid jets - a data driven modelling approach, Exp. Therm. Fluid Sci. 76, 109 (2016). 